\begin{tabular}{|c|l|}
\hline Title & Photoluminescence in implanted and doped silicon near room temperature \\
\hline Author(s) & $\begin{array}{l}\text { Matsubara, Ichiro; Sasahara, Shingo; Mishina, Tomobumi; Ishibashi, Y asuhiko; Kobay ashi, Toshihiko; Nakahara, } \\
\text { Jun'ichiro }\end{array}$ \\
\hline Citation & $\begin{array}{l}\text { physica status solidi (b), 243(8), 1893-1897 } \\
\text { https://doi.org/L0.1002/pssb.200541220 }\end{array}$ \\
\hline Issue Date & 2006-05-02 \\
\hline Doc URL & http://hdl.handle.net/2115/10506 \\
\hline Rights & Published in physica status solidi (b), 243(8), 1893-1897, 2006. \\
\hline Type & article (author version) \\
\hline File Information & pss.pdf \\
\hline
\end{tabular}

Instructions for use 


\title{
Photoluminescence in implanted and doped silicon near room temperature
}

\author{
Ichiro Matsubara*1, Shingo Sasahara ${ }^{1}$, Tomobumi Mishina ${ }^{1}$, \\ Yasuhiko Ishibashi ${ }^{1,2}$, Toshihiko Kobayashi ${ }^{2}$, and Jun'ichiro Nakahara ${ }^{1}$ \\ ${ }^{1}$ Division of Physics, Hokkaido University, Sapporo 060-0810, Japan \\ ${ }^{2}$ Department of Electrical and Electronics Engineering, Kobe University, Kobe 657-8501, Japan
}

\begin{abstract}
Key words Silicon, photoluminescence, indirect, phonon assisted PACS 04A25
\end{abstract}

Photoluminescence in implanted and doped silicon at room temperature is measured, and the observed structures are assigned as intrinsic-phonon-assisted indirect allowed transitions. The temperature of photoexcited carriers, which are higher than the bath temperature, is estimated. For confined carriers produced by boron implantation the temperature dependence of the effective temperature of the excited carriers is same for the different samples, but an enhancement of the photoluminescence is observed.

Copyright line will be provided by the publisher

\section{INTRODUCTION}

Investigations on semiconductors have shifted to topics related to nanotechnology recently. The development of detectors in the near-infrared region have stimulated the investigation of the basic optical properties of Si near room temperature. There have been almost no detailed measurements of the temperature dependence of photoluminescence (PL) in Si crystals, exhibiting an indirect optical transition at high temperatures (around room temperature), because of the weakness of the PL. Recently, electroluminescence (EL) was observed by Homewood et al.[1], and PL was investigated by Prins et al. [2] and by Ishibashi et al.[3] in boron implanted Si. Photoluminescence enhancement in EL devices was explained as follows: near the boron-implanted surface of Si, the dislocation loops introduce a local strain field, that modifies the band structure and provides spatial confinement of charge carriers in the non-defective region near the surface. It is considered that this spatial confinement allows room temperature EL at the band-edge owing to the very clean surface region of Si. Such devices are highly compatible with ULSI (ultra large scale integration) technology, as boron ion implantation is already routinely used in silicon device fabrication.

In this B-implanted $\mathrm{Si}$, and $\mathrm{n}(\mathrm{p})$-type doped $\mathrm{Si}$, we observe PL spectra that we attribute to phonon-assisted recombination of free excitons. Thorough investigations of PL for indirect forbidden transitions was reported in thallous halides $[5,6]$ but for $\mathrm{Si}$ it has so far proved difficult to measure PL at high temperatures. In this paper, we observe intrinsic phonon-assisted photoluminescence, an enhancement in the phonon-assisted indirect transitions in p- and n-type doped $\mathrm{Si}$, and an abnormal increase in electron temperature and PL intensity of photoexcitation especially in B-implanted Si.

* Corresponding author: I. Matsubara e-mail: ichirou@phys.sci.hokudai.ac.jp, Phone: +81 117064426 , Fax: $+81117064426$ 


\section{EXPERIMENTS}

Commercial n- and p-type Si as a substrate and B-implanted Si[1] are used for PL observation. Excitation light for PL are focused on the Si surface, at wavelengths of $488 \mathrm{~nm}$ or $514.5 \mathrm{~nm}$ from a $30 \mathrm{~mW}$ Ar ion laser. The PL is collected on a $25 \mathrm{~cm}$ monochromator and a photomultiplier (Hamamatsu Photonics R5509-41) sensitive to $1.4 \mu \mathrm{m}$ radiation. The sensitivity of the system is calibrated using black body radiation. At low temperatures PL intensities have been observed to increase at the absorption edges ' $2 \mathrm{a}$ ' and ' $1 \mathrm{a}$ '[4]. We observe no impurity-associated PL in n- and p-type commercial Si above $20 \mathrm{~K}$.

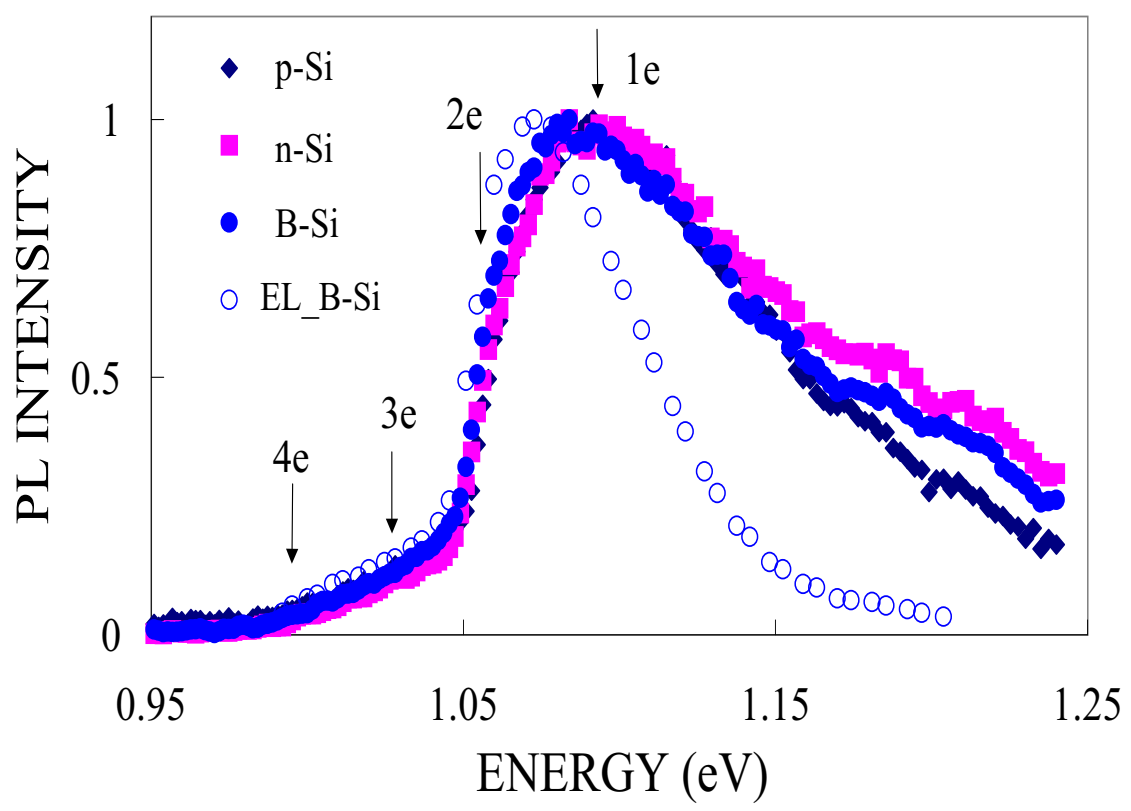

Fig. 1 Normalized photoluminescence of different Si samples at 300K. Only phonon-assisted indirect transitions are observed. The closed diamonds, closed squares, and closed circles correspond to commercial p-type $\mathrm{Si}$ (p-Si), n-type $\mathrm{Si}(\mathrm{n}-\mathrm{Si})$, and in B-implanted $\mathrm{Si}$ (B-Si) at room temperature, respectively. The intensities of the electroluminescence are shown by open circles. (EL_B-Si) are the data reproduced from the figure in Ref. [1].

At higher temperatures PL spectra are decomposed into three components for phonon-assisted indirect transitions as in the following Eq.(1):

$$
I \propto \sum_{i} B_{i}(T) \sqrt{E-E_{i}} \exp \left(-\left(E-E_{i}\right) / k_{B} T_{e}\right)
$$

The threshold ' $2 \mathrm{e}$ ' of the PL corresponds to ' $2 \mathrm{a}$ ' in the absorption spectra, and the lower energy thresholds ' $3 \mathrm{e}$ ' and ' $4 \mathrm{e}$ ' of the PL correspond to the absorption edges ' $3 \mathrm{a}$ ' and ' $4 \mathrm{a}$ ' of Ref. [4], as shown in Fig. 3. The line shape of the absorption is fitted by this equation. Here $T_{e}$ is the thermalized temperature of the excited electrons near the band edges of the conduction and valence bands, and $B_{i}(T)$ is a slowly varying function of temperature. The agreement between the observed and calculated line shapes is very good, and typical examples of both spectra are shown in Fig.2. 


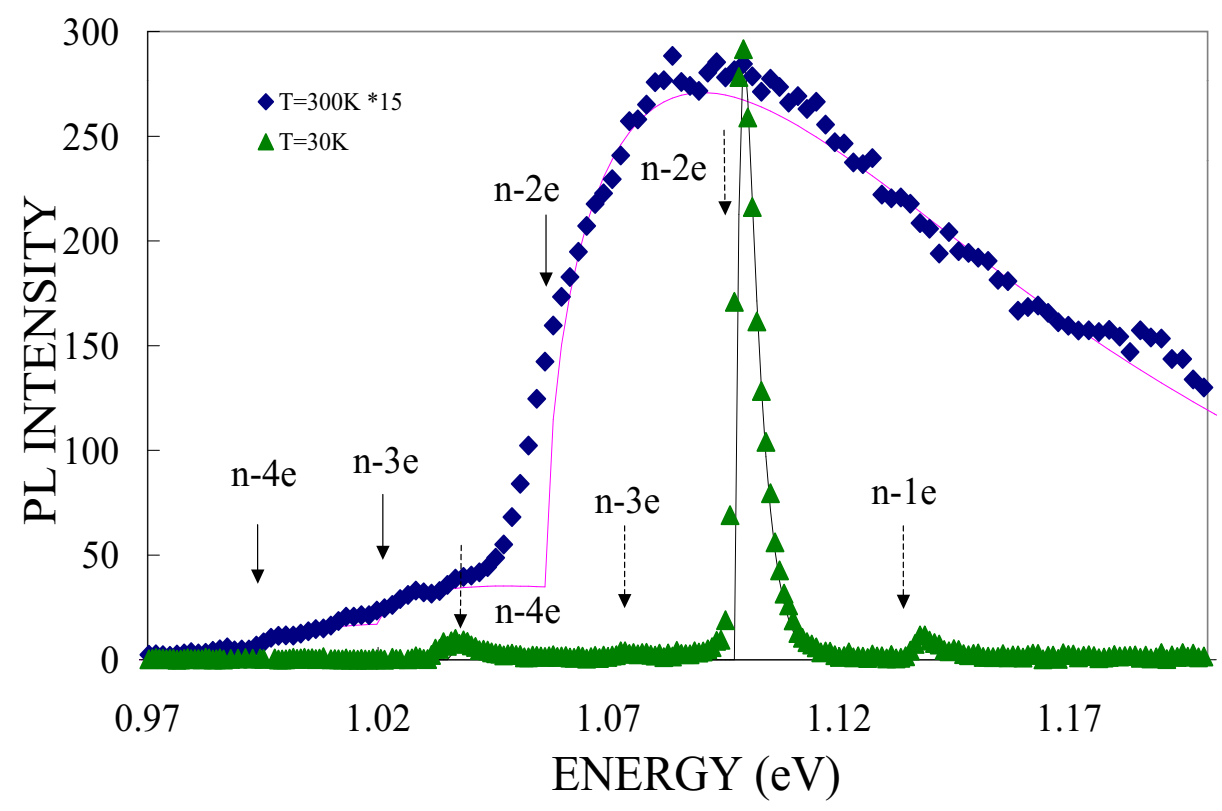

Fig. 2 Photoluminescence spectra at 30K (triangles) and 300K (diamonds) in n-Si and their fitted line shape using Eq.(1).

In Fig. 3 solid lines show the threshold energies of phonon-assisted absorption spectra [4], and the broken lines correspond to phonon emission processes and phonon absorption processes at high temperatures [4]. Symbols labeled as n-, p- and B- indicate the threshold energies corresponding to the different Si sample, and the labels ' $\mathrm{e}$ ' and ' $\mathrm{a}$ ' refer to phonon emission and absorption, respectively. At low temperatures, PL thresholds are observed at four absorption thresholds ' $1 \mathrm{e}$ ', '2e', '3e', and '4e', whose temperature dependences are plotted in Fig.3. The intensities of all PL peaks decrease with increasing temperature below $180 \mathrm{~K}$ for $\mathrm{n}-$ and $\mathrm{p}-\mathrm{Si}$. Above $200 \mathrm{~K}$, the PL with the threshold at ' $2 \mathrm{a}$ ' increases with temperature, following the increase in phonon creation probability. This increase is smaller than that observed for the PL of B-implanted Si. Thus the thresholds labelled as 1, 2, 3 and 4 correspond to different phonon emission processes in PL. The labels ' $a$ ' and 'e' respectively indicate the phonon absorption and emission processes for both absorption spectra and PL. The labels ' $a$ ' and 'e' for PL and absorption threshold energies are opposite for the same energy.

\section{DISCUSSIONS}

The temperature dependence of PL intensity is fitted by the following equation for the phonon emission process:

$$
B_{i}(T) \propto \frac{1}{1+a \exp \left(-\Delta E / k_{B} T_{e}\right)}\left[n\left(\hbar \omega_{i}\right)+1\right] .
$$

Here $n\left(\hbar \omega_{i}\right)$ is the population of phonons with energy $\hbar \omega_{i}, \Delta E$ is the barrier height for transitions from excited states to nonradiative centres, and $T_{e}$ is the temperature of thermalized carriers in excited states. Here the determined parameters $\Delta E / k_{B}$ and $a$ using Eq.(2) are, respectively, $260 \mathrm{~K}$ and 350 in p-Si and $200 \mathrm{~K}$ and 130 in n-Si. But the observed enhancement for B-implanted Si is 


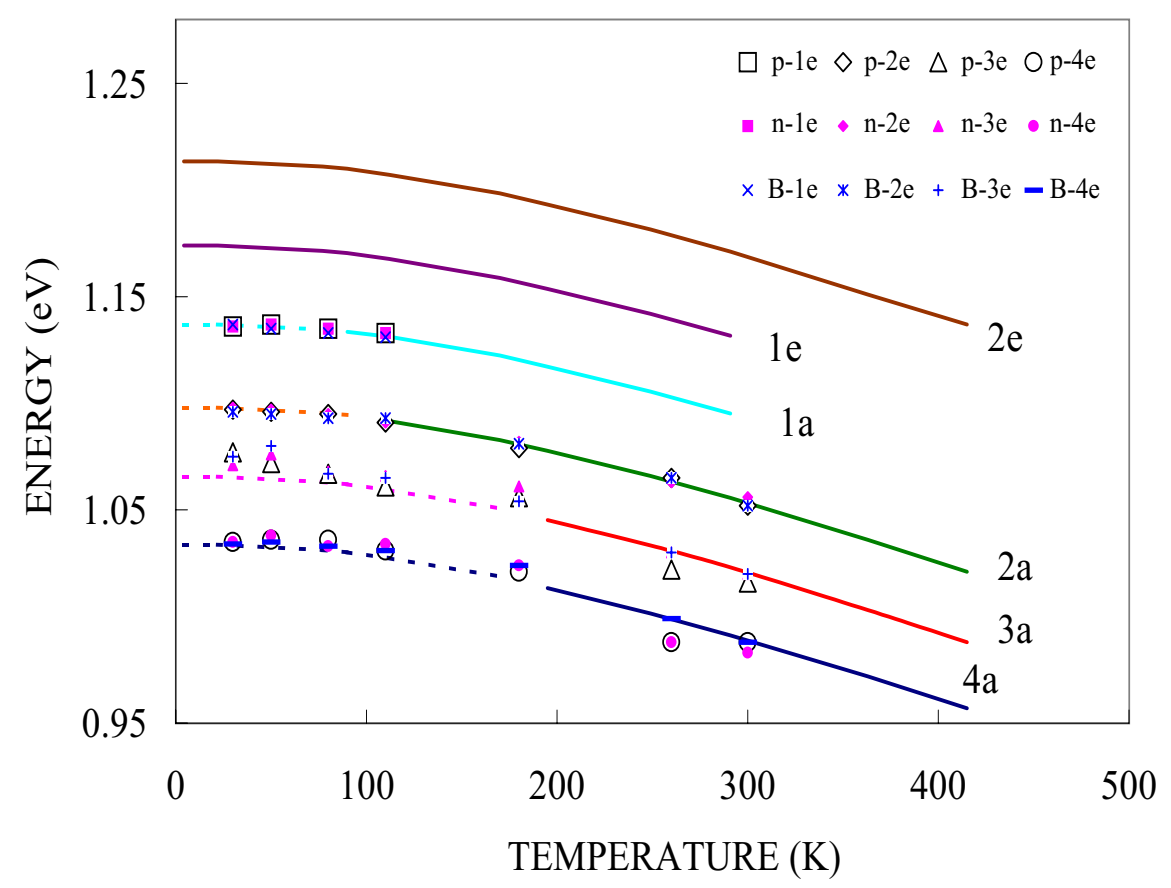

Fig. 3 Threshold energies of the absorption spectra (lines [4]) and of the PL spectra (see key for the symbols corresponding to p-type $\mathrm{Si}, \mathrm{n}$-type $\mathrm{Si}$ and B-implanted Si.). Open symbols, closed symbols, and the others correspond to p-type $\mathrm{Si}, \mathrm{n}$-type $\mathrm{Si}$, and B-implanted $\mathrm{Si}$ for phonon emission processes, respectively.

more than twice that calculated by Eq.(2). This temperature dependence is related to the increase in the lifetime [7]. High carrier temperatures are much higher than the bath temperature (dotted straight line), but the temperatures of excited carriers are almost the same for all the different types of Si, as shown in Fig.4. The excited carriers are not thermalized with the lattice. Further, the thermalized temperature of the excited carriers is almost independent of the intensity of optical excitation. These results indicate that the excess energy of excited electrons corresponds to the difference $\left|\Delta E_{g}\right|$ between the energy gap at temperature $\mathrm{T}$ and at absolute zero. The detailed mechanism for the temperature dependence of the PL intensity is not known. As is clear from Fig. 5, PL intensities increase a little near room temperature for commercial n- and p-Si following Eq.(2) for phonon-assisted indirect transitions. But the PL enhancement is very large in the case of B-implanted Si compared to the ratio 1.1 calculated from Eq.(2) for the corresponding transition, in accordance with the change in phonon creation probability between $50 \mathrm{~K}$ and $300 \mathrm{~K}$. The temperature dependence of the threshold ' $2 \mathrm{e}$ ' of the PL thresholds is the same as the threshold '2a' previously observed [4]. Likewise for the estimated positions at low temperatures for the other absorption thresholds, as shown in Fig. 3. The observed PL line shape coincides with the EL spectra previously observed [1], as shown in Fig. 1 by the open squares. The EL threshold energes are the same as the thresholds of our PL spectra. This indicates that the lifetime only becomes longer in B-implanted Si at high temperatures. In Fig. 5 an enhancement of the intensity above that expected from the variation in phonon creation probability near room temperature is observed only in B-implanted $\mathrm{Si}$, whereas an enhancement according to the variation of the phonon population alone is sufficient to explain the data for the other samples. The internal 


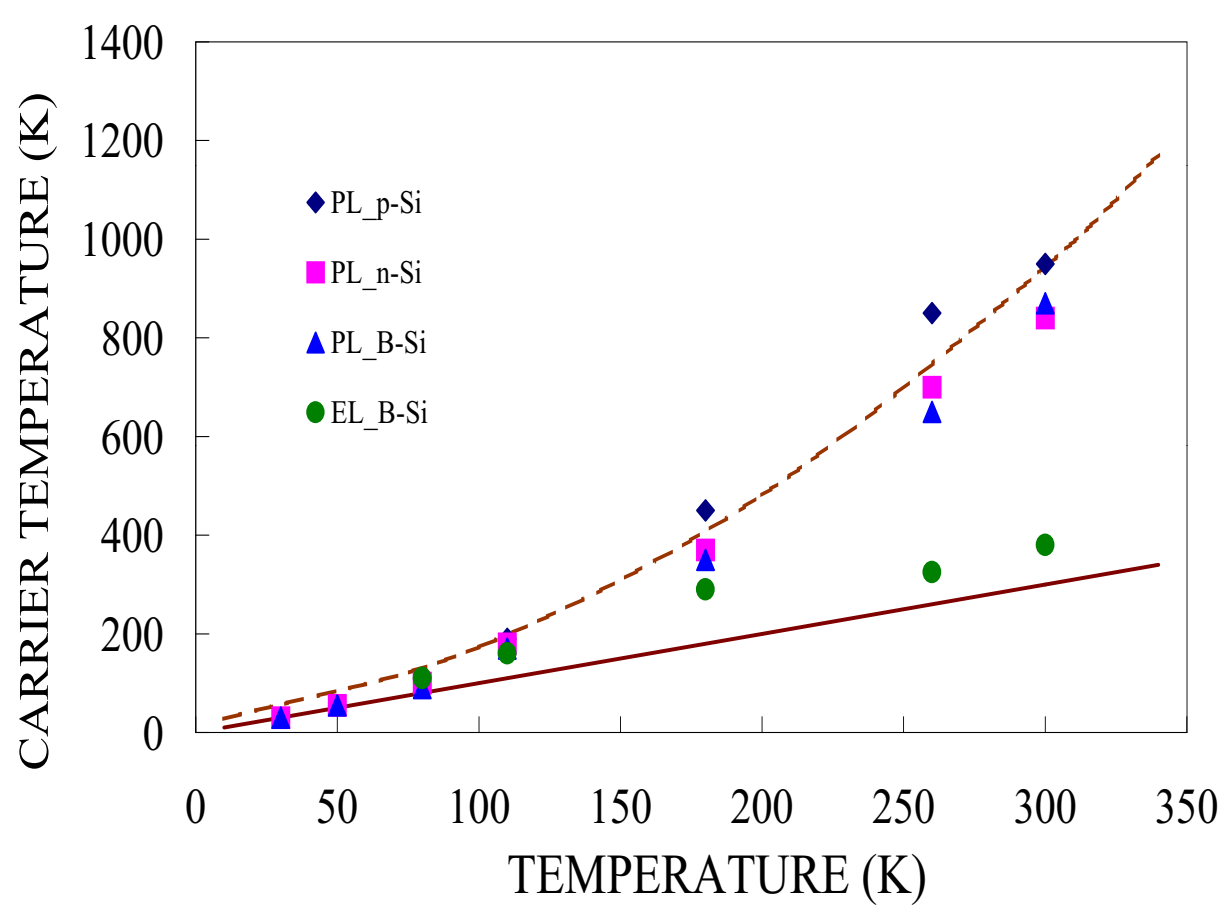

Fig. 4 Effective electron temperature. The straight line indicates the same temperature. The symbols indicate the carrier temperatures calculated using Eq.(1) in the line shape fitting. The curved line indicates $\left|\Delta E_{g}\right| / k_{B}+T$. Here, $\left|\Delta E_{g}\right| / k_{B}$ is the change of the energy gap with respect to $0 \mathrm{~K}$. The agreement between the curved line and the calculated effective electron temperature variation indicates that this change in energy gap corresponds to the excess energy (under energy relaxation of excited carriers to the bottom of the band) expressed in Kelvin, and that the excited carriers are not thermalized with the lattice except for the case of the EL of B-implanted Si.

quantum efficiency can be written as $\eta_{i}=R_{B B} /\left(R_{S R H}+R_{B B}+R_{A u g e r}\right)$, where $R_{B B}$ is the radiative recombination rate, $R_{\text {Auger }}$ is the Auger recombination rate, $R_{S R H}=\Delta n / \tau_{S R H}$ is the multiphonon recombination rate owing to deep levels in the band gap. Here $\tau_{S R H}$ is increased as the temperature increases, and $\eta_{i}$ becomes larger when $\tau_{S R H}$ becomes longer [7]. In B-implanted $\mathrm{Si}, \tau_{S R H}$ is promoted by the gathering action of the implanted boron as in the case of phosphorous [7] and the PL enhancement is larger than that expected from the variation in the phonon creation probability.

\section{CONCLUSION}

The temperature dependence of the PL spectra in differently doped and implanted Si sample is observed from room temperature to low temperatures $\sim 20 \mathrm{~K}$. Near the absorption edge, the various structures in the PL spectra can be assigned to different phonon creation and annihilation indirect transitions. The temperature of the thermalized excited carriers is higher than the temperature of the heat bath by an amount equal to the excess energy, which is almost same as the energy difference $\left|E_{g}(T=0)-E_{g}(T)\right|$ from the indirect gap of Si estimated from optical absorption data. 


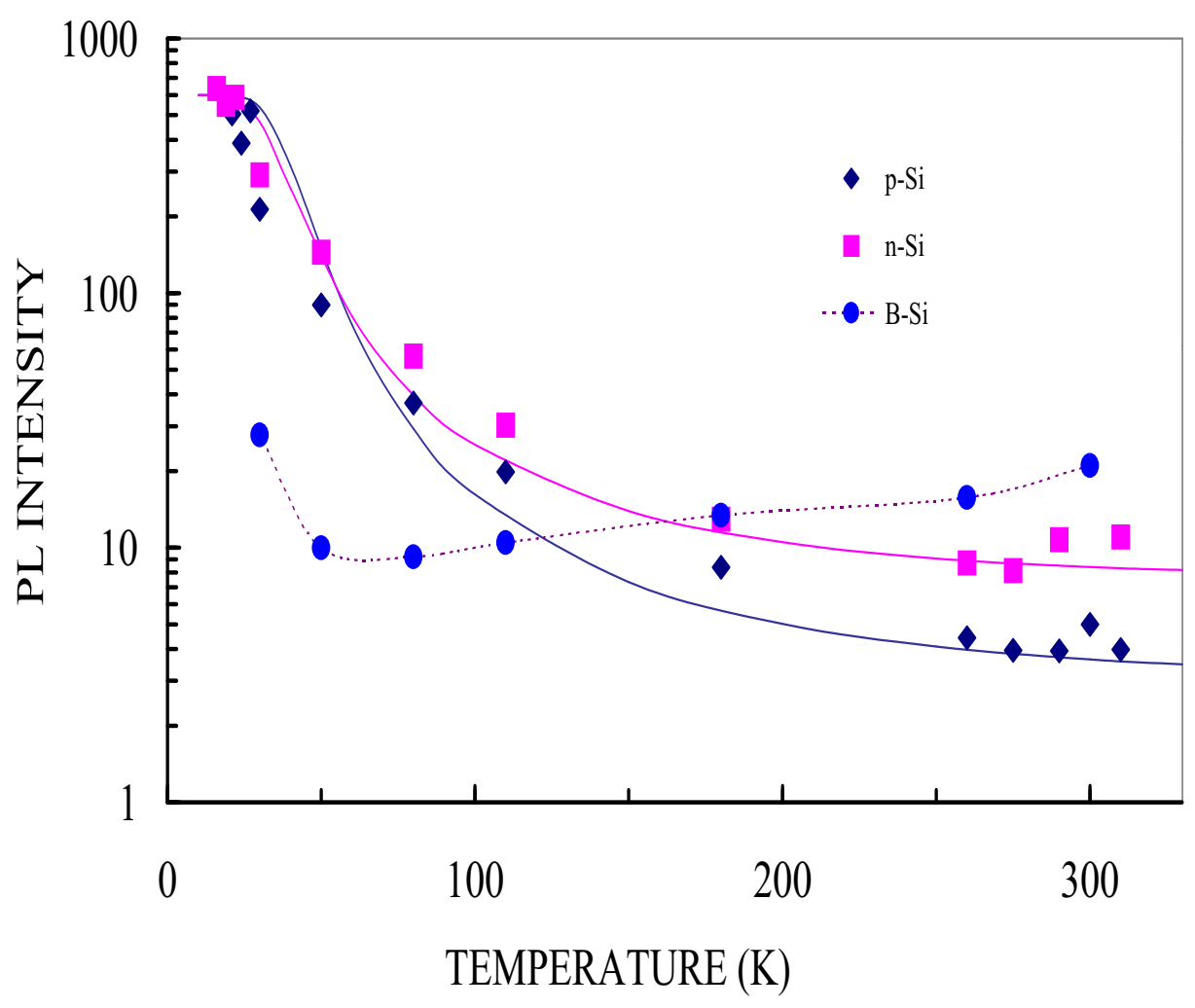

Fig. 5 Temperature dependence of the PL intensity in p-Si (closed diamonds), n-Si (closed squares) and B-implanted Si (closed circles). The solid lines are fitted curves using Eq.(2). The dotted line is a guide for the eye to the PL data of B-implanted Si. (Because of the abnormal temperature dependence we provide no fitting curve.

Acknowledgements The authors acknowledge to Profs. A. Prins, and K. P. Homewood for technical assistance and discussions, and to Dr. S. Yamamoto for providing samples and making measurements.

\section{References}

[1] W. L. Ng, M. A. Lourenco, R. M. Gwilliam, S. Ledain, G. Shao, and K. P. Homewood, NATURE, $410192(2001)$

[2] A. D. Prins, Y. Ishibashi, S. Sasahara, J. Nakahara, M. A. Lourenco, R. M. Gwilliam, T. Kobayashi, A. Nagata, and K. P. Homewood, phys. stat. sol. (b) 241, No. 14, 3387-3390 (2004)

[3] Y. Ishibashi, S. Sasahara, A. Nagata, T. Sugimoto, A. D. Prins, T. Kobayashi and J. Nakahara, Rev. High Press. Sci. \& Tech. 14, 93 (2004) in Japanese.

[4] G. G. Macfarlane, T. P. McLean, J. E. Quarrington, and V. Roberts, Phys. Rev. 111, 1245-1254 (1958)

[5] J. Nakahara, A. Fujii, K. Takiyama and K. Kobayashi, J. Phys. Soc. Jpn. 37, 1312-1318(1974)

[6] A. Fujii, J. Nakahara, K. Kobayashi and Y. Fujii, J. Phys. Soc. Jpn. 46, 1218-1224(1979)

[7] M. Kittler, T. Arguirov, A. Fischer and W. Seifert, Optical Materials 27, 967-972(2005) and the references. 